\title{
The Profile of Secondary Metabolites of Sponge Clathria reinwardtii Extract as a Result of Fe Accumulation in Spermonde Archipelago
}

\author{
Lydia Melawaty ${ }^{1}$, Kristiana Pasau ${ }^{2}$ \\ ${ }^{1}$ Chemical Engineering Department, Paul Christian University of Indonesia, Makassar, Indonesia \\ ${ }^{2}$ Mechanical Engineering Department, Paul Christian University of Indonesia, Makassar, Indonesia \\ Email: Iylamety@yahoo.com, kristianapasau@yahoo.co.id
}

Received 12 August 2015; accepted 27 December 2015; published 30 December 2015

Copyright (C 2015 by authors and Scientific Research Publishing Inc.

This work is licensed under the Creative Commons Attribution International License (CC BY).

http://creativecommons.org/licenses/by/4.0/

(c) O) Open Access

\begin{abstract}
Study on the effect of Fe metal concentrations in Spermonde Archipelago on secondary metabolites production from sponge was conducted. The percent of peak area and $m / z$ from GC-MS at $t_{R}=$ 13.555 were affected by accumulated Fe in Chlathria reinwardtii, where at high Fe concentration a lower percent peak area was produced compared to Clathria reinwardtii extract at low Fe concentration. Chromatogram CR-LL-DCM produced 2 peaks at $t_{R}=21.401$ and 25.174 which were not observed in CR-BC-DCM chromatogram. This indicated that Clathria reinwardtii in Lae-Lae island produced metabolite secondary compounds that were not produced by Clahtria reinwardtii in Barrang Caddi island. FT-IR analysis indicated that Clathria reinwardtii accumulating Fe with highest and lowest concentration produced strong peaks at wavenumber of about $3400 \mathrm{~cm}^{-1}$ for $0-\mathrm{H}$ and $\mathrm{N}-\mathrm{H}$ stretches. $\mathrm{O}-\mathrm{H}$ pliable and $\mathrm{C}-\mathrm{O}$ stretch peaks at wavenumber of about $1200 \mathrm{~cm}^{-1}$ had changes from medium intensity at low Fe concentration (CR-BC-DCM) to weak at high Fe concentration (CR-LL-DCM).
\end{abstract}

\section{Keywords}

Clathria reinwardtii, Fe, GC-MS, FTIR

\section{Introduction}

The water environment of Spermonde Archipelago is an abundant source of bioactive compounds. Bioactive

How to cite this paper: Melawaty, L. and Pasau, K. (2015) The Profile of Secondary Metabolites of Sponge Clathria reinwardtii Extract as a Result of Fe Accumulation in Spermonde Archipelago. Advances in Biological Chemistry, 5, $266-272$. http://dx.doi.org/10.4236/abc.2015.57023 
compounds from marine environment are primarily in the form of secondary metabolite compounds with great potential to be developed as medicine materials. Sponges produce various bioactive secondary metabolite chemical compounds. Specific chemical compounds are produced to defend themselves from predators, to bind their soft body, and settled. Secondary metabolite compounds are natural chemical substances source that can be found in nature and can be made as reference for medicines development and to support various industrial interests. These substances will never be depleted and continuously produced with interconverted molecular structure from time to time.

Ecologically, sponges occur at various habitat conditions. This poses a question regarding the adaptation mechanism of sponges, which are simple organism, toward their habitat environment conditions. Studies suggest that sponges have a chemical self-defense (secondary metabolite). These chemical compounds are useful to defend themselves from competitor pressure, antagonism reaction, infection or predation by other marine organisms [1]. Sponges produce two types of metabolites during their growth and development, namely primary metabolites and secondary metabolites. Primary metabolites are metabolites formed during growth period and used in metabolism processes that are essential to organism. Whereas, secondary metabolites are compound components produced when primary metabolite requirements have been met and used in evolutionary mechanism of the species or adaptation strategy to environment [2]. The characteristics of secondary metabolites include the formation of secondary metabolites that is highly influenced by the environment conditions of the organism and each of the secondary metabolites are produced by only some certain organisms [3] [4].

Secondary metabolite compounds play important roles in important biological processes including reproduction, competition, and protection against predators [5] [6]. Sponges have highly diverse compounds in their tissues and this has an effect to predators, competitors, community structure and dynamic, and evolution [7] [8]. Understanding the causes of this variation is a valuable information not only about factors affecting the secondary metabolite production but also conditions where sponges invest more on chemical protection.

Essential metals in biota cells are required not only for growth but also for self-protection from predators. Fe is required by sponges to synthesize secondary metabolites. The influence of heavy metal pollution can increase or decrease the secondary metabolites production in sponges.

Water environment conditions in Spermonde Archipelago due to low or high Fe concentration will influence the formation of secondary metabolites in the sponge. Heavy metal Fe and biota cells are needed not only for growth but also for self-defense against predator using the produced metabolites. The effect of Fe pollution can increase or reduce the secondary metabolite production in sponge at Spermonde Archipelago region.

\section{Method}

Clathria reinwardtii samples were chopped, and then dried under indirect sunlight for 3 days and crushed with blender. Sponge powder was macerated by methanol (Merck) solvent for $1 \times 24$ hours three times. Maceration result was separated from residu by decantation and then filtered by Whatman 41 paper. Organic macerates were taken and the solvent was evaporated by using rotavapour Buchi.

Methanol extract was then partitioned with mixture of dichloromethane (Merck) solvent and water at 1:1 ratio. Extraction was performed until the mixture was clear. The obtained dichloromethane extract was collected and then analyzed by FT-IR Shimadzu model IR Prestige-21 and GC-MS Agilent Technologies 7890A.

\section{Results and Discussion}

GC-MS result of dichloromethane extract from sponge Clathria reinwardtii provided chromatograms as can be seen in Figure 1. GC chromatograms shows retention time difference and percentage of peak area of compound components in each of dichloromethane extract from Clathria reinwardtii with highest and lowest Fe concentration. The same is true for compound fragmentation indicated by different Mass Spectrophotometry spectrum for each of the dichloromethane extract from the same sponge species but different in accumulated Fe concentration.

Both of these chromatograms show a peak with 99\% similarity to $\alpha$-limonen (compound from data center) and have a very high percentage of peak area at retention time $\left(t_{R}\right)=13.555$, namely $62.82 \% \mathrm{with} \mathrm{m} / \mathrm{z} 136.1$ (CR-LL-DCM) and 90.32\% with $\mathrm{m} / \mathrm{z}=136.1$ (CR-BC-DCM) (Figure 2). Data on percentage of peak area and $\mathrm{m} / \mathrm{z}$ at $t_{R}=13.555$ was influenced by Fe accumulated in Clathria reinwardtii where at high Fe concentration the percentage of peak area was lower than Clathria reinwardtii extract at lower Fe concentration.

CR-LL-DCM chromatogram produced 2 peaks at $t_{R}=21.401$ and 25.174 which were not found in chro- 
mate-gram CR-BC-DCM. This indicated that Clathria reinwardtii in Lae-Lae islands produced secondary metabolite compounds that were not produced by Clathria reinwardtii in Barrang Caddi islands as a mechanism to protect themselves against hi pollutant Fe concentration either in the Clathria reinwardtii theirself or in the sediment.

Compound produced by CR-LL-DCM at $t_{R}=21.401$ (Figure 3) had 38\% similarity to compound 1,1-dimethyl thiourea with percentage of peak area $=13.37$ and $\mathrm{m} / \mathrm{z}$ value $=405$. Similarity level was very low due to different fragmentation pattern and $m / z$ value of the compound from data center was 1,1-dimethyl thiourea $=104$.
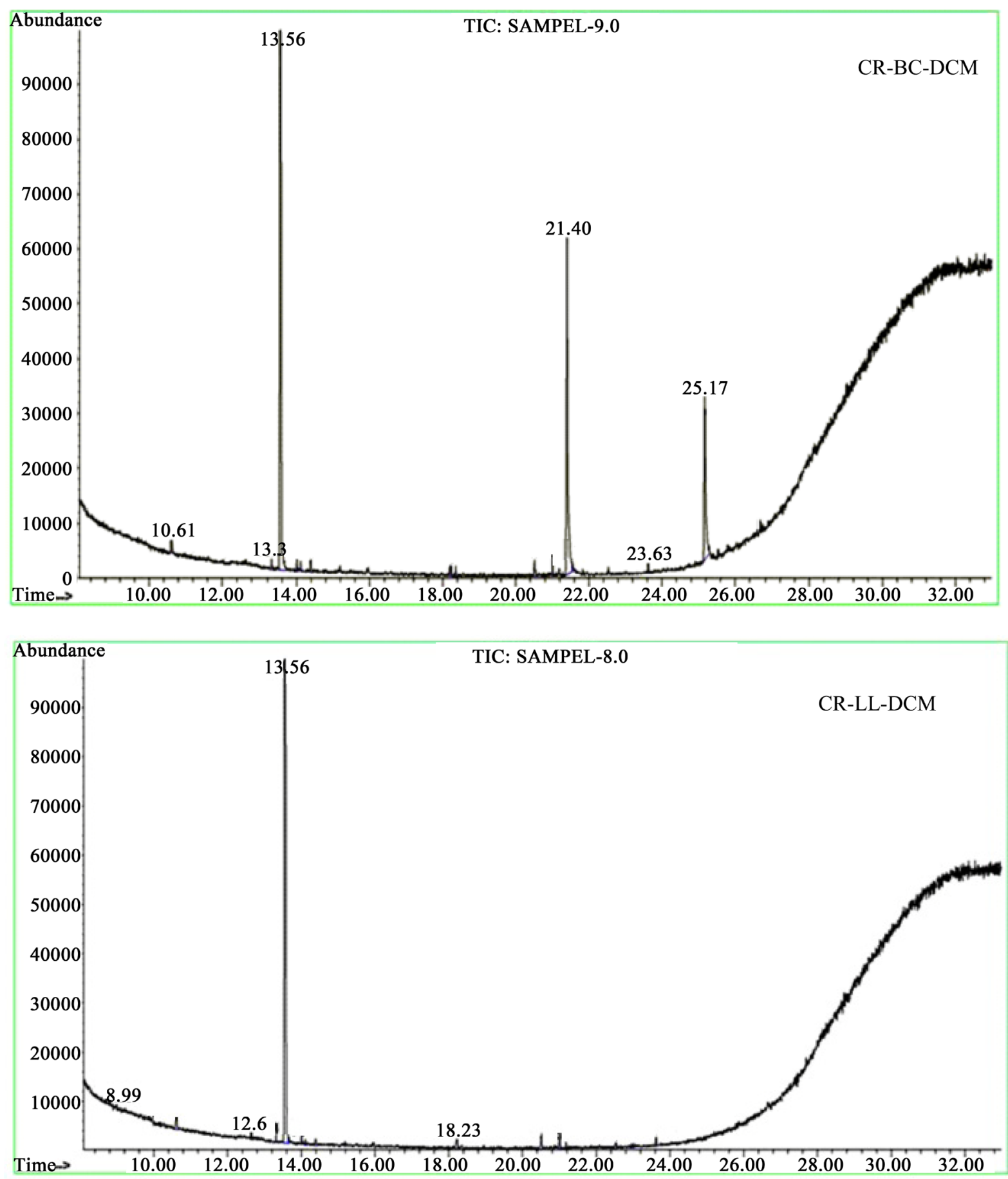

Figure 1. GC-MS chromatograms of dichloromethane extract of Clathria reinwardtii. 


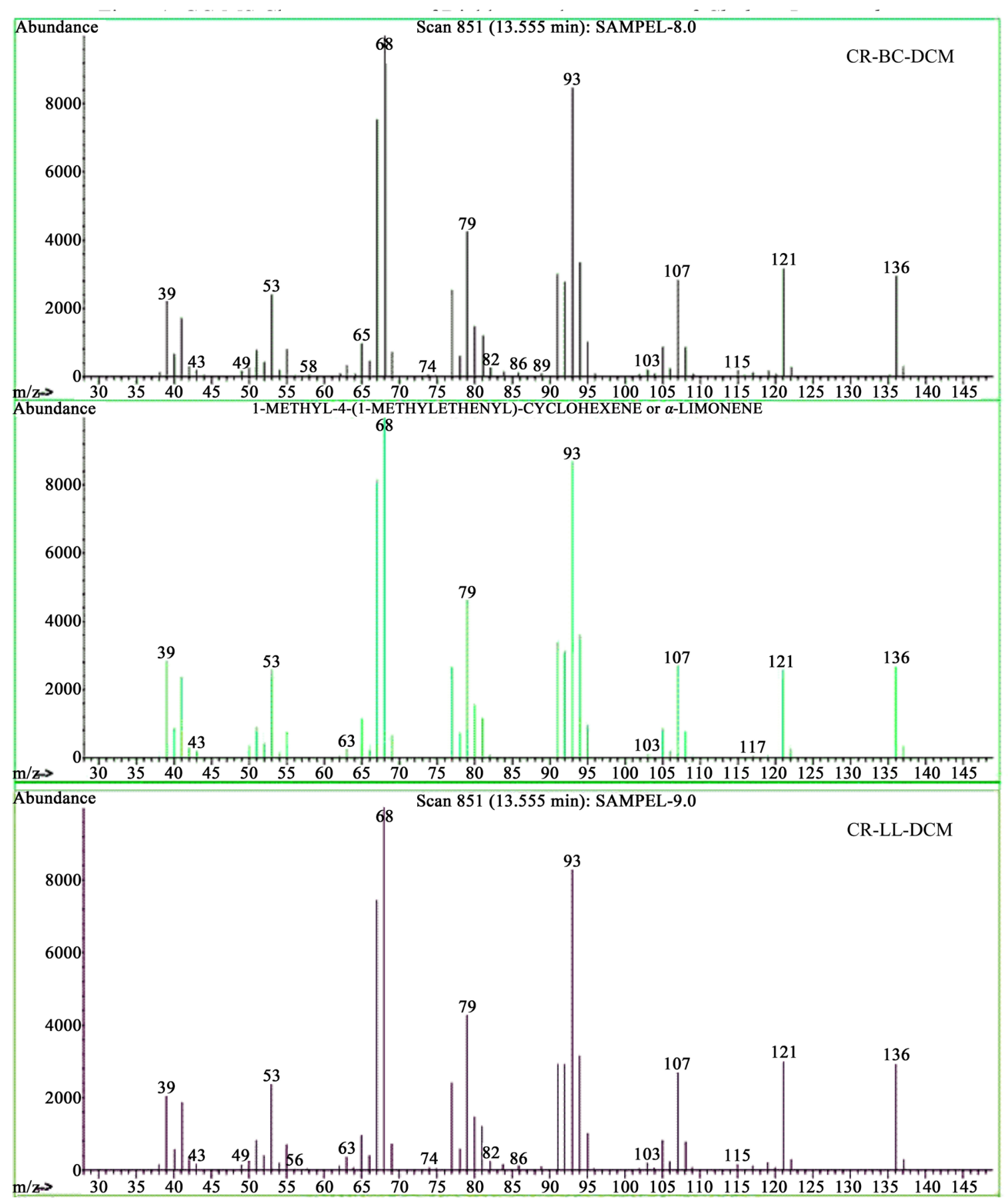

Figure 2. Mass spectrophotometry spectra at $t_{R}=13,555$ of Clathria reinwardtii.

The compound at $t_{R}=25.174$ (Figure 4) had 83\% similarity to the compound (1-methyl ethyl)-2-[(aminocarbonil) oxylmethyl]-2-methyl penthyl ester carbamate with percentage of peak area $=12.04$ and $\mathrm{m} / \mathrm{z}$ value $=281$. The similarity level was only $83 \%$ because the fragmentation pattern is not fully equal and the $\mathrm{m} / \mathrm{z}$ value from the central data was (1-methyl ethyl)-2-[(aminocarbonil) oxylmethyl]-2-methyl penthyl ester carbamate $=260$.

FT-IR analysis results as shown in Figure 5 indicates that Clathria reinwardtii accumulating Fe at highest and lowest concentration produced strong peak at wave number of about $3400 \mathrm{~cm}^{-1}$ for $\mathrm{O}-\mathrm{H}$ and $\mathrm{N}-\mathrm{H}$ stretch. Flexible peak $\mathrm{O}-\mathrm{H}$ and $\mathrm{C}-\mathrm{O}$ stretch at wave number of about $1200 \mathrm{~cm}^{-1}$ subjected to change from medium intensity at low Fe concentration (CR-BC-DCM) to weak intensity at high Fe concentration (CR-LL-DCM). 


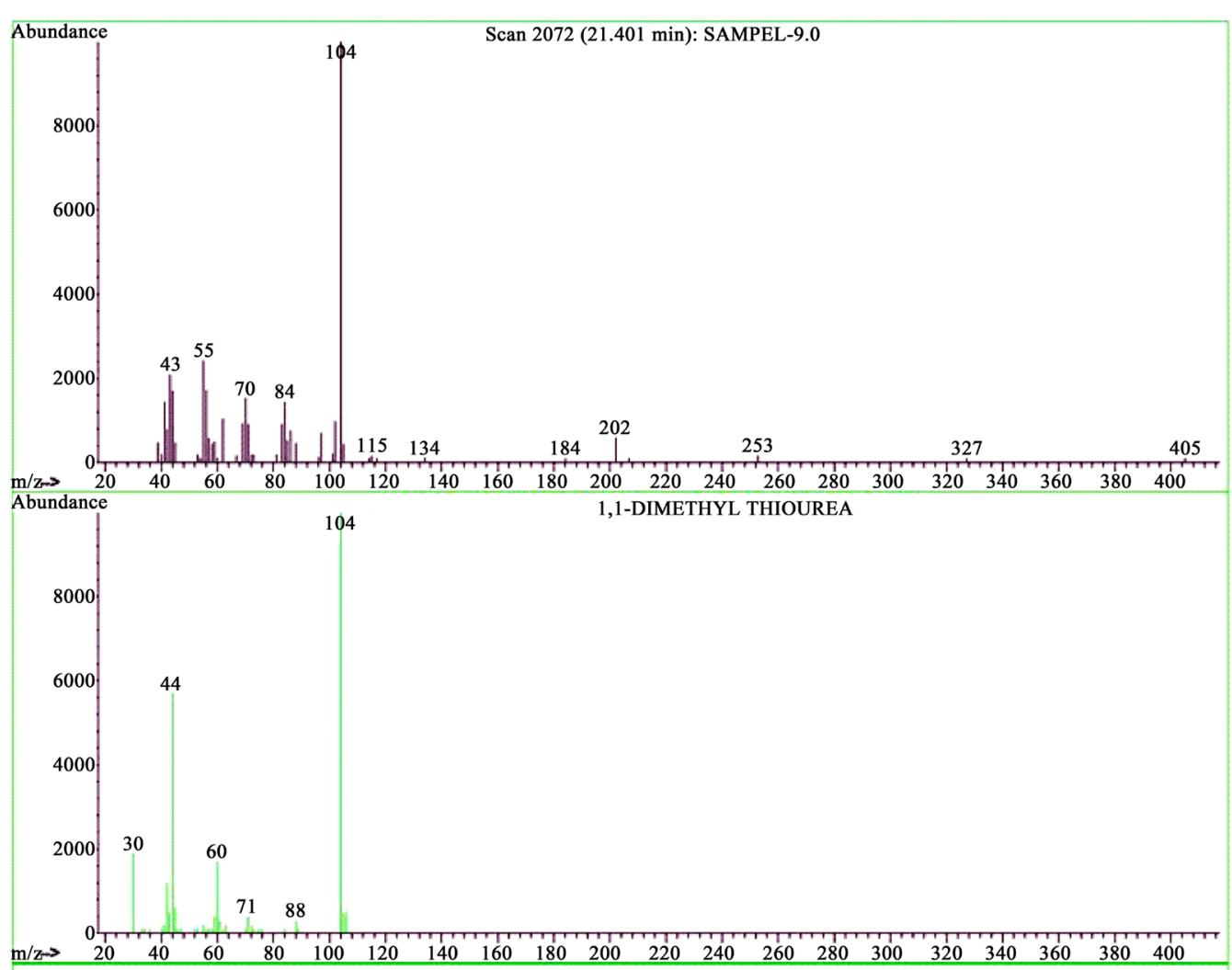

Figure 3. Mass spectrophotometry spectra at $t_{R}=21,401$ of Clathria reinwardtii (CR-LL-DCM).

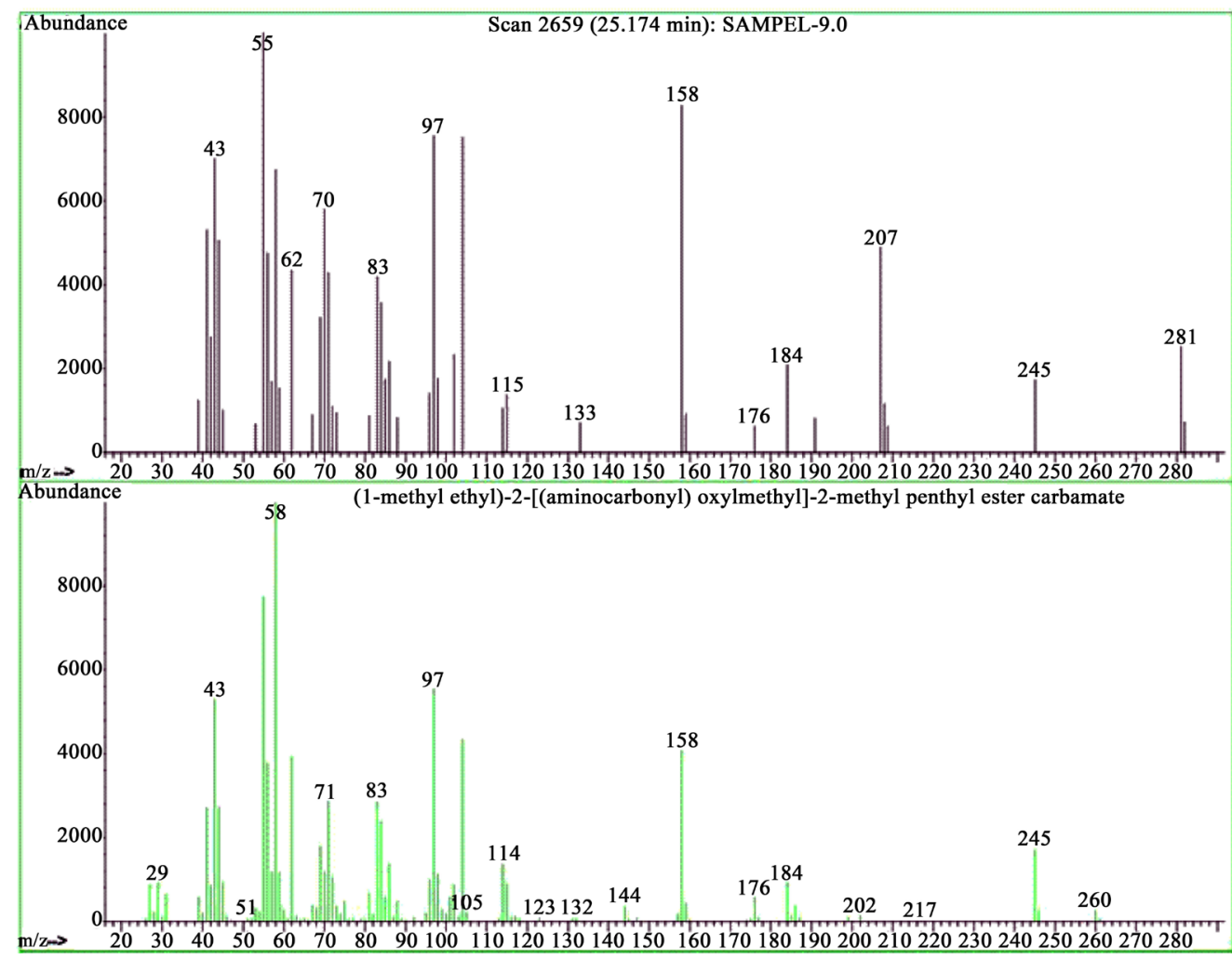

Figure 4. Mass spectrophotometry spectra at $t_{R}=25,174$ from Clathria reinwardtii (CR-LL-DCM). 


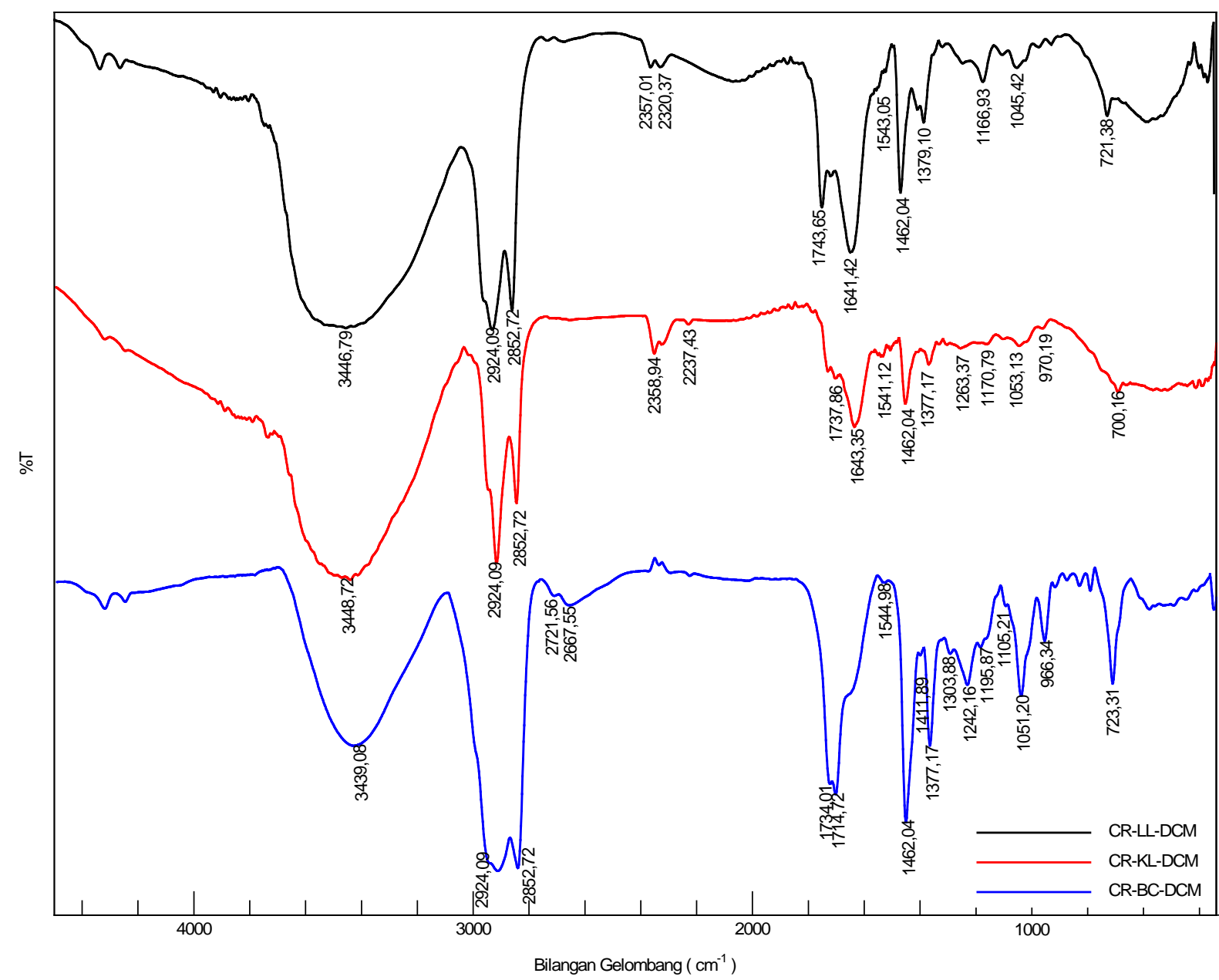

Figure 5. FT-IR spectrum of Clathria reinwardtii secondary metabolites.

The change in intensity of O-H flexible peak and C-O stretch from medium to weak indicated the influence of Fe at high concentration resulting the weakening of $\mathrm{O}-\mathrm{H}$ flexible and $\mathrm{C}-\mathrm{O}$ stretch peaks. These peaks underwent shift of $21 \mathrm{~cm}^{-1}$ to bigger wave number direction or lower wavelength (blue shift). The wave number shift can be caused by induction, mesomery effect, and field effect [9]. Peak at $1240.23 \mathrm{~cm}^{-1}$ (CR-LL-DCM) underwent only change to low intensity. The change in absorption intensity from strong to medium or from medium to weak indicated that the secondary metabolite concentration decreased in Clathria reinwardtii extract due to the effect of high Fe concentration.

The data obtained from measurements results of dichloromethane extract of Clathria reinwardtii using GC-MS and FT-IR indicated that the influence of Fe accumulated in high concentration on secondary metabolites that can be produced. The obtained molecular weight from Mass Spectrophotometry for dichloromethane extract of Clathria reinwardtii with high Fe concentration was generally greater than extract with low Fe concentration.

\section{Conclusions}

Chromatograms indicated peaks with $99 \%$ similarity to $\alpha$-limonen with percentage of peak area at retention time $\left(t_{R}\right)=13.555$ where at high Fe concentration produced percentage of peak area $62.82 \%$ and at low Fe concentration $90.32 \%$.

Chromatograms indicated that Clathria reinwardtii at $t_{R}=21.401$ and 25.174 in Lae-Lae island produced secondary metabolite compounds that were not produced by Clathria reinwardtii in Barrang Caddi island as a mechanism of self defense against high Fe concentration in the Clathria reinwardtii itself or in sediment. 
The change in intensity of O-H flexible and C-O stretch peak from medium to weak indicated the influence of $\mathrm{Fe}$ at high concentration that weakened the O-H flexible and C-O stretch capability. These peaks underwent shift of $21 \mathrm{~cm}^{-1}$ to the direction higher wave number or lower wavelength (blue shift).

\section{References}

[1] Thoms, C. and Schupp, P.J. (2007) Chemical Defense Strategies in Sponges: A Review. Porifera Research Biodiversity, Innovation, and Sustainability, Rio de Janeiro, 627-637.

[2] Torssel, K.B.G. (1983) Natural Product Chemistry. A Mechanisitc and Biosynthetic Approach to Secondary Metabolism. John Wiley and Sons Ltd., New York.

[3] Crueger, W. and Crueger, A. (1982) Biotechnology: A Textbook of Industrial Microbiology. Science Tech. Inc., Madison.

[4] Madigan, M.T., Martinko, J.M., Parker, J. and Brock, T.D. (2000) Biology of Microorganisms. Prentice-Hall Inc., Upper Saddle River.

[5] McClintock, J.B and Baker, B.J. (2001) Marine Chemical Ecology. CRC Press, Boca Raton.

[6] Paul, V.J and Puglisi, M.P. (2004) Chemical Mediation of Interactions among Marine Organisms. Natural Product Reports, 21, 189-209. http://dx.doi.org/10.1039/b302334f

[7] Cimino, G. and Ghiselin, M.T. (2001) Marine Natural Products Chemistry as an Evolutionary Narrative. In: McClintock, J.B. and Baker, B.J., Eds., Marine Chemical Ecology, CRC Press, Boca Raton, 115-154.

[8] Paul, V.J., Cruz-Rivera, E. and Thacker, R.W. (2001) Chemical Mediation of Macroalgal-Herbivore Interactions: Ecological and Evolutionary Perspectives. In: McClintock, J.B. and Baker, B.J., Eds., Marine Chemical Ecology, CRC Press, Boca Raton, 227-265.

[9] Supratman, U. (2010) Elucidation of Organic Compound Structures. Spectoscopy Method for Organic Compound Structure Determination. Widya Padjadjaran. 